\title{
THE NEXT GENERATION OF GERMANIUM SUBSTRATES: EXPOGERTM
}

\author{
Pieter Arickx $^{(1)}$, Rufi Kurstjens ${ }^{(1)}$, Wim Geens ${ }^{(1)}$, Kristof Dessein ${ }^{(1)}$ \\ (1) Umicore Electro-Optic Materials, Watertorenstraat 33 - B2250 Olen (Belgium), \\ Email: pieter.arickx@eu.umicore.com
}

\begin{abstract}
Umicore has been the leading Germanium substrate manufacturer over the last 20 years. We are only able to maintain that position by constantly improving the quality of our product. In this work, Umicore wants to present how it will continue this tradition by adding even more value to its product.

In the beginning of 2014 we proposed to our customers to help them in achieving their yield improvement goals. Two main concerns were expressed: the breakage rate in their production line and non-uniform epigrowth. After two years of development, Umicore has bundled these two improvements into a new product. The ExpoGer ${ }^{\mathrm{TM}}$ wafer combines a better mechanical yield with a uniform, epi-ready surface enabling a narrower distribution of the electrical performance.
\end{abstract}

The breakage rate of the customers is related to the wafers that suffer from early failure. The percentage of early failures is related to its thickness. With the trend towards even thinner substrates, it is important to add features that reduce the percentage of early failures. Two features are crucial: the shape of the substrate edge and the stress relief etch, which influences the wafer strength.

Just as for the strength, two distinctive aspects define the surface quality. On the one hand, there are the number and size of surface defects, which have an important influence on the metal-organic chemical vapour deposition (MOCVD) growth performed at Umicore's customers. On the other hand, drying marks, which are invisible to the naked eye, may lead to a local, non-uniform growth and they can be a precursor to time dependent haze issues.

Umicore is able to demonstrate the impact of the ExpoGer ${ }^{\mathrm{TM}}$ processes on the strength and surface quality of its wafers and thus on the production yield of its customers.

\section{INTRODUCTION}

Germanium substrates are mainly used as the epitaxial growth substrate of high-efficiency, triple junction solar cells for space applications. Staying true to Umicore's value proposition, we developed specific improvements that increase the Ge substrate quality significantly. By focusing on our customer's wishes, we were able to create a new product: the ExpoGer ${ }^{\mathrm{TM}}$ wafer.

When our customers perform the MOCVD growth to create the triple junction solar cell, any deviation from a perfect surface will be a nucleation point for a defect or a cause of a non-uniform epi-stack. These imperfections can be caused by surface roughness, crystal originated pits (COPs), particles or non-uniform layers of native oxide. The area and density of these imperfections will determine the impact on the growth and the final solar cell performance.

The improvements focus on avoiding the larger area and higher density imperfections. Through adjusting the cleaning and drying, the unwanted surface roughness, irregular oxide thickness, particles and drying marks could be avoided. Additionally, the adjusted clean also reduces the impact of COPs. The formation of COPs during crystal pulling was not addressed in this work.

Wafer breakage occurs due to brittle fracture. The stressed material does not deform plastically and it fails in the elastic regime with very little absorption of energy. Small chips or cracks can be the nucleation site for a fracture if enough stress is applied on that specific location. To avoid these defects, two processes have been updated. First, the edge now has a more rounded profile, making it more robust towards impact and the process itself is inherently less prone towards chipping. Secondly, these microscopic cracks on the etched surface are avoided by using a newly developed etch in a very controlled environment. The roughness of the ExpoGer ${ }^{\mathrm{TM}}$ wafer is significantly lower than our current standard wafers and thus has less nucleation sites for a fracture.

This paper will show our first, internal results for 100 $\mathrm{mm}$ substrates. Umicore is currently transferring these developments to $150 \mathrm{~mm}$ as well.

\section{MECHANICAL STRENGTH}

\subsection{Wafer Edge}

When the edge of a wafer has sharp corners, stress is concentrated in these points. Geometric discontinuities cause an object to experience a local increase in the intensity of a stress field. Examples of shapes that cause these concentrations are cracks, sharp corners, holes, and changes in the cross-sectional area of the object. High local stresses can cause objects to fail more 
quickly, so engineers must design the geometry to minimize stress concentrations. During processing, upon impact with the edge, chipping or wafer fracture will occur more easily for sharp edges. To avoid this stress concentration, the wafer edge can be bevelled or round.

Chen et al [1,2] have compared 4 types of edge profile, schematically represented at the bottom of Figure 1. They have tested the breakage energy necessary to induce wafer breakage by designing a drop test where a $200 \mathrm{~g}$ weight is dropped from some height on the edge of the wafer. The recorded breakage energy is seen to increase as the edge is more and more rounded.

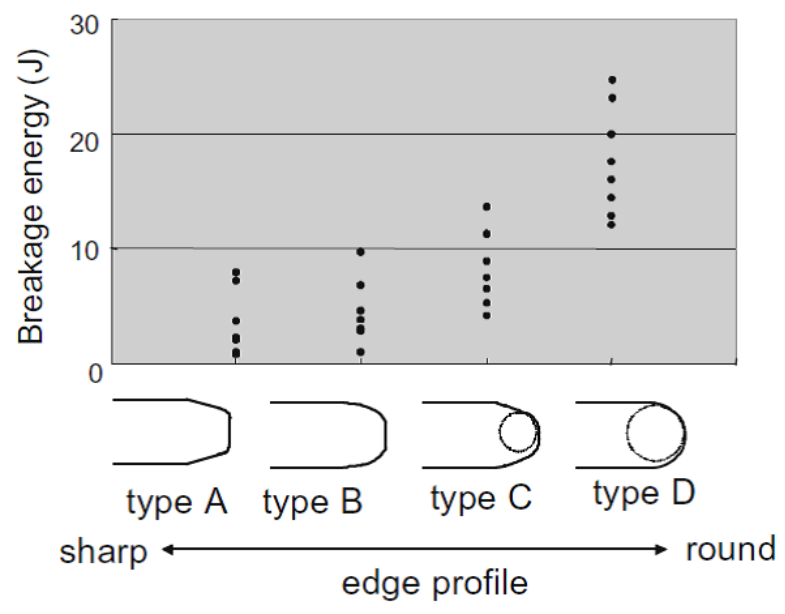

Figure 1. The results of a mechanical drop test for four kinds wafer edge profile. [1,2]

Chen et al then compared these experimental results to wafer breakage ratios for the four types of edges from a production environment. The breakage ratio of 10000 wafers processed with each type of edge design is shown in Figure 2. It can be seen that the breakage ratio drops from $3400 \mathrm{ppm}$ to roughly $1200 \mathrm{ppm}$ when going from the edge with sharp corners to the round edge.

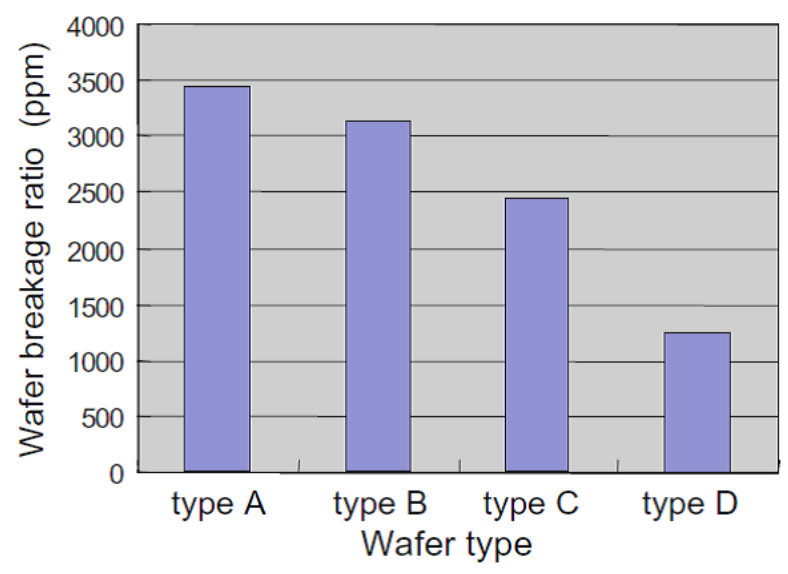

Figure 2. The wafer breakage ratio of type A to type $D$

\section{in a production line tested on 10000 wafers. [1,2]}

Guided by the above, we were able at Umicore to obtain the rounded edge profile for our wafers. Not only was the edge grinding wheel modified to fit these new requirements, the entire process flow needed to be carefully reviewed as not all steps are perfectly symmetrical, i.e. the removal and the front side of the wafer is not necessarily equal to the removal on the back side (e.g. only the front side is polished).

In Figure 3 the profile of the rounded edge can be seen.

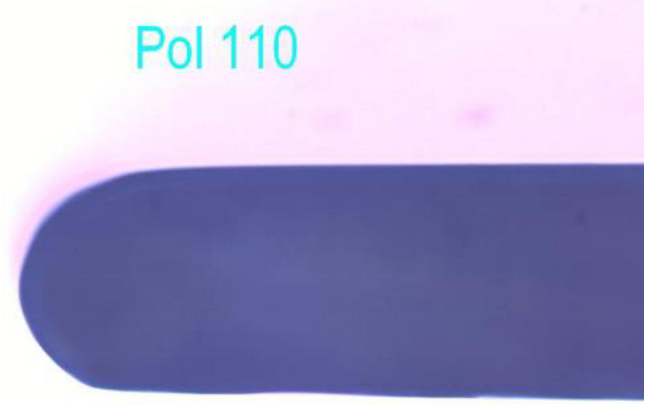

Figure 3. Round edge profile of a polished wafer along the (110) crystal direction.

\subsection{Wafer Surface}

The stress relief etch immediately follows the mechanical processing steps of the wafering process, as this step needs to remove all the mechanically induced damage from the previous steps, such as wafer sawing and surface grinding. The subsurface damage (SSD) is inversely related to the breakage strength, as can be seen in Eq. 1:

$$
\sigma_{f}=\frac{K_{I c}}{\sqrt{\pi a}}
$$

where $K_{I c}$ is the stress intensity factor (in MPa.m ${ }^{1 / 2}$ ), $\sigma_{f}$ is the fracture stress and a is the crack length.

As the breakage strength is related to the crack length, it is clear that the SSD needs to be removed to obtain the strongest substrate possible, while maintaining the geometrical characteristics of the wafer. Furthermore, the surface needs to remain smooth, as uncontrolled etching would induce deeper defects which could serve as crack nuclei. The etchant thus needs to be designed to meet these requirements. 
The fracture strength of the wafer is measured by the ball-on-ring method (Instrom uniaxial set-up) where the wafer rests on a ring with an internal diameter smaller than the wafer itself. This technique withholds edge defects such as chipping or small cracks and only tests the structural strength of the surface. When the ball (with a diameter of $5 \mathrm{~mm}$ ) brings down a certain force on the surface, the opposite side experiences a strain force. The unpolished backside experiences this strain force as the wafers are always placed with the polished side up. The ball is gradually lowered $(0.01 \mathrm{inch} / \mathrm{min})$ until the strain breaks the wafer. The breaking load is usually expressed as pounds-force per square inch (lbf). Typically, breaking loads are distributed according to a Weibull distribution.

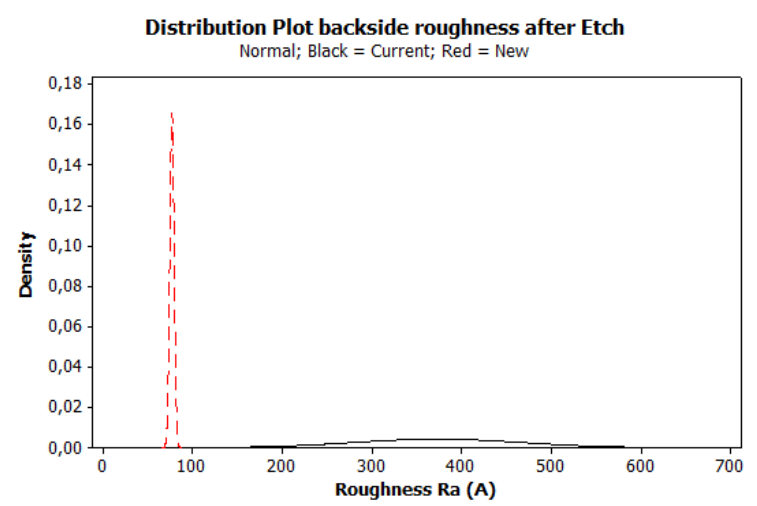

Figure 4. The roughness of the etched (back) side of the wafer of the current (black) and the new (red) process.

Umicore has replaced the current, stress relief etch process, by a newly developed etch process that improves the surface related strength of the wafer. Due to a highly controlled process, the roughness stays well below the roughness of our current substrates (Figure 4) and the average strength of $175 \mu \mathrm{m}$ (final thickness) substrates almost doubles, as clearly demonstrated in Figure 5.

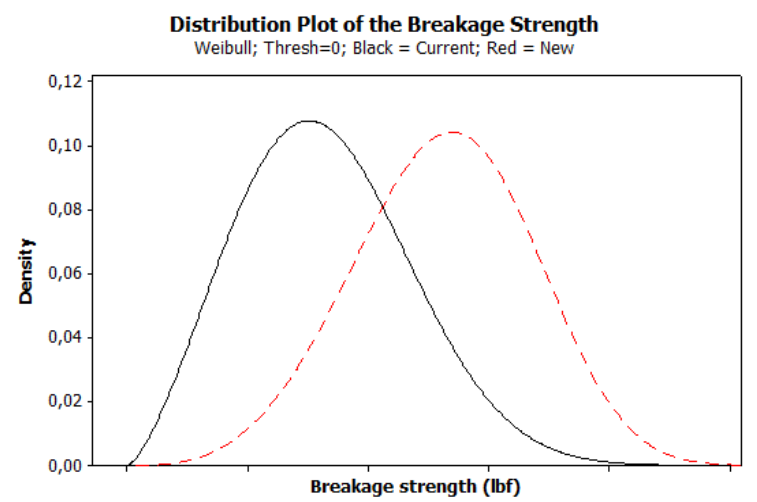

Figure 5. The breakage strength the wafer etched with the current (black) and the new (red) process.
Both improvements act as an enabler for the storage and transportation of thin $100 \mathrm{~mm}$ substrates in multi-wafer shippers (MWS).

\section{Surface Quality}

\subsection{An Alternative Clean}

The final clean process has an impact on the size of the defects present on the wafer surface. The purpose of the new clean was to drastically alter the behaviour with respect to the defects and uniformity of the wafer process while maintaining the cleaning efficiency. If the defects or uniformity variations on the wafer surface can stay below a certain threshold, they will not act as initiation sites for MOCVD growth defects.

The Umicore final clean holds two major parts: a particle clean to remove any leftover abrasive from the polishing step and a metal clean to remove trace metals from the wafer surface.

The particle clean is evaluated via the particle removal efficiency. We have developed a method to intentionally contaminate our substrates with the particles used as the abrasive in the polishing process. We measure before and after deliberate contamination and after the clean. Figure 6 is a demonstration of this method, measured by a KLA-Tencor Candela, a surface inspection system.

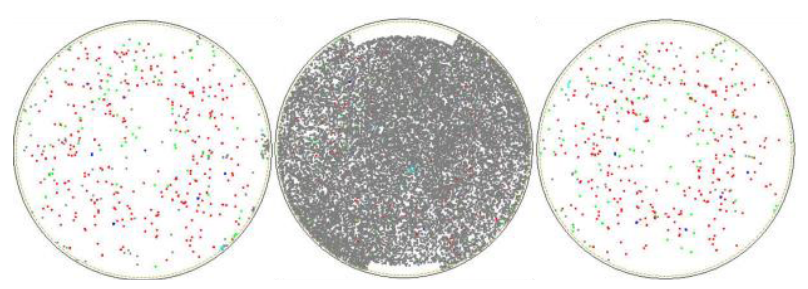

Figure 6. A clean, contaminated and a re-cleaned wafer (from left to right) measured by a KLA-Tencor Candela.

The particle removal efficiency (PRE) can be calculated as shown in Eq. 2.

$P R E=\left(1-\frac{\text { Particles }_{\text {AftClean }}-\text { Particles }_{\text {BefCont }}}{\text { Particles }_{\text {AftCont }}-\text { Particles }_{\text {BefCont }}}\right) \cdot 100$

As shown in Figure 7, the PRE of the new clean is $100 \%$. 


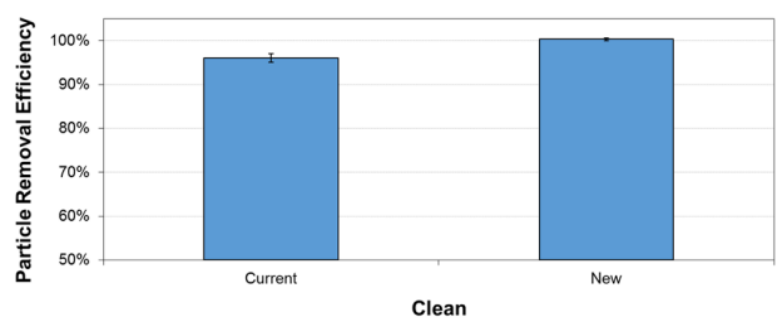

Figure 7. The particle removal efficiency of the current and the new clean process.

The main feature of the new clean is of course its neutral behaviour towards the crystallographic properties and uniformity of the wafer. Crystallographic features on the substrate surface will not be altered. Figure 8 shows that a substrate processed via the standard process shows more surface defects. Both wafers were sampled adjacent to each other from the crystal. The standard clean causes a larger degradation of the final surface made "visible" by the KLA-Tencor Candela tool.

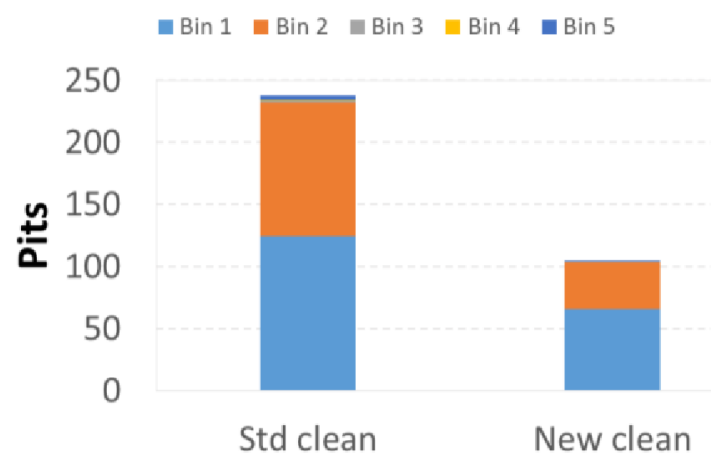

Figure 8. The occurrence of surface anomalies on the substrate's surface depending on the final clean binned as a function of the anomaly size with smallest elements in bin 1 and the largest elements in bin 5 .

Trace metals are detrimental for triple junction solar cells and should thus be avoided at all time. A clean was developed with the same metal removal capabilities, while having less impact on the quality of the polished surface. Figure 9 demonstrates that for a myriad of metals, the new clean is similar or even better than the current clean. These measurements were done by TXRF, where the metals were etched by HF from the substrate surface.

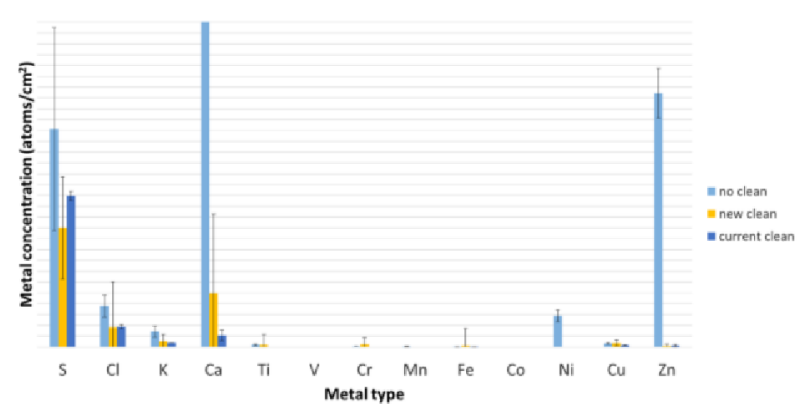

Figure 9. The occurrence of metals on the substrate's surface as a function of the final clean.

\subsection{New Drying Technology}

As described in the introduction, the occurrence of drying marks will lead to growing defects and in a further stage can even support the formation of haze on the wafer surface. The marks itself are created when water is allowed to evaporate on the wafer surface. The normal drying process uses the Marangoni effect to prevent this from happening. To further improve the drying of wafers, Umicore recently acquired a new tool where the entire process was redesigned, albeit still being based on the Marangoni effect.

In the current process, the contact area between the wafers and the supporting combs of the dryer is still significant. When the alignment of the wafer into the comb is not perfect, water can be trapped between the comb and the wafer and evaporate on the surface, leaving a drying mark, as shown in Figure 10.

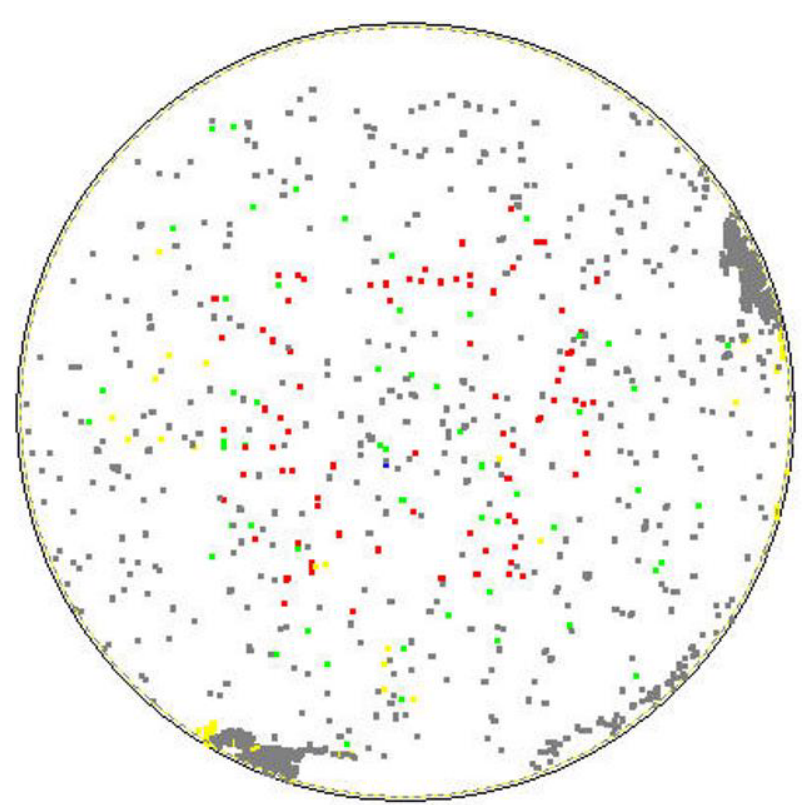

Figure 10. The grey areas at the sides of the wafer indicate drying marks.

The new process has a minimal contact area between the wafer and the positioning comb and special features 
are in place to avoid misalignment and minimize the chance of water being trapped between comb and wafer. This allowed Umicore to reduce the occurrence and the impact of drying by evaporation, instead of the Marangoni effect drying. Figure 11 shows a KLATencor SurfScan image, another surface inspection method, where the drying marks of the new tool are indicated. It is clear that these are minimal compared to the current process.

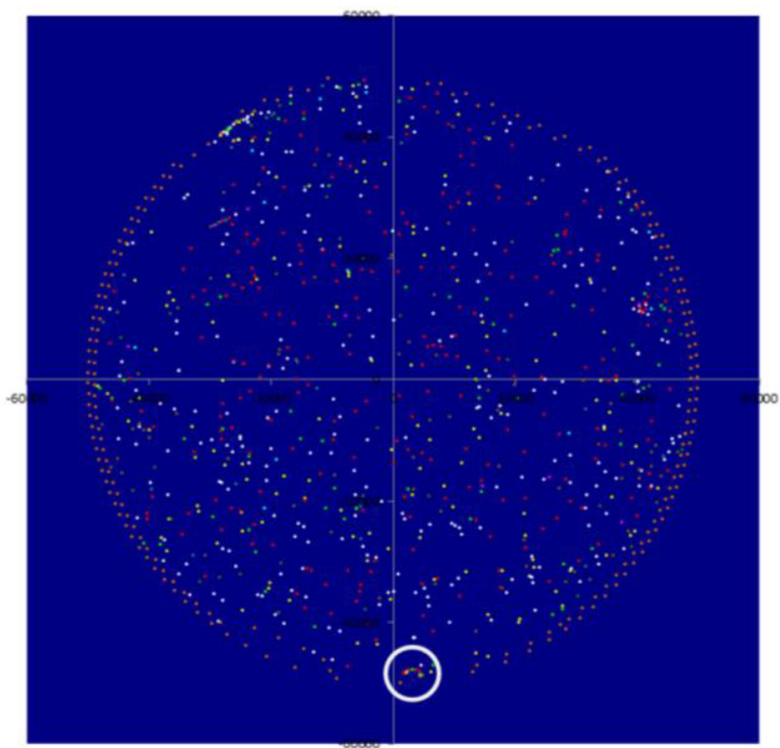

Figure 11. The circled area at the side of the wafer indicates a drying mark.

\section{CONCLUSIONS}

It has been demonstrated that the new generation of Umicore Germanium substrates are both stronger and show a promising improvement of the surface quality. With the ExpoGerTM wafer, Umicore addresses two main concerns of its customers.

The stronger substrate was obtained by redesigning the edge grinding process and the stress relief etch, for the best combination of an impact-resistant edge with a smooth and strong substrate back side. These improvements enable transportation in MWS, which in turn enable automatic handling of wafers.

The quality of the surface was improved by developing new particle and metal cleans, while at the same time improving the drying process at the end of the cleaning.

\section{ACKNOWLEDGEMENTS}

The authors would like to thank the European Space Agency (ESA) for the funding of the project with Contract No 4000110920/14/NL/CBi/fk (ESAImager III).

\section{REFERENCES}

1. P.-Y. Chen, M.-H. Tsai, W.-K. Yeh, M.-H. Jing, \& Y. Chang. (2010). Relationship between wafer edge design and its ultimate mechanical strength. Microelectronic Engineering, vol. 87, no. 11, pp. 2065-2070.

2. P.-Y. Chen, M.-H. Tsai, W.-K. Yeh, M.-H. Jing, \& Y. Chang. (2010). Relationship between wafer fracture reduction and controlling during the edge manufacturing process. Microelectronic Engineering, vol. 87, no. 10, pp. 1809-1815. 